\title{
Recent Advancements in 3D X-ray Microscopes for Additive Manufacturing
}

\author{
Leah Lavery $^{1}$, William Harris ${ }^{1}$, Jeff Gelb ${ }^{1}$ and Arno Merkle ${ }^{1}$. \\ 1. Carl Zeiss X-ray Microscopy, Pleasanton, CA, United States of America.
}

Three-dimensional X-ray microscopy (XRM) is a powerful sub-surface imaging technique that reveals tomography of three-dimensional microstructure from a range of materials, non-destructively. The nondestructive nature of X-rays has made the technique widely appealing, with the potential for characterizing sample changes in "4D," delivering 3D microstructural information on physically the same sample over time, as a function of sequential processing conditions or experimental treatments. This has led to a new generation of functional studies with applications and is in a state of rapid expansion [1]. Recently, laboratory-based X-ray sources have been coupled with high resolution X-ray focusing and detection optics from synchrotron-based systems to acquire tomographic datasets with resolution down to $50 \mathrm{~nm}$ across a great span of sample dimensions [2]. This signifies an improvement of at least one order of magnitude in spatial resolution relative to the limits of 'optic-free' laboratory computed tomography (CT) techniques. This talk will explore both the implementation of optics in nanoscale and sub-micron laboratory XRM architectures and review in detail several leading applications examples for the field of additive manufacturing. XRM tomographic data provides multiscale imaging and visualization for a wide variety of $\mathrm{AM}$ materials such as 3D characterization of scaffold implant materials, porosity quantification in sintered powder steels, even creation of accurate 3D-printed models in biomimetic studies [3-4].

Additive manufacturing (AM) techniques can produce complex 3D structures. The reliability and performance of the produced parts relies heavily on the resultant microstructure. Frequently the material performance can be quite sensitive to its discrete, complex pore structure, thereby motivating the need to investigate and understand the morphology of these materials in 3D at the appropriate pore scale. XRM provides a means to perform non-destructive 3D characterization of complex even anisotropic geometries. Beyond imaging, quantification of volumetric properties such as porosity can be calculated from the reconstructed dataset. This is highly dependent on the spatial resolution of the imaging system. Porosity is an important property because for materials destined for high stress applications should be fully-dense to avoid failure in service. XRM has been used to characterize porosity in metal additive process control studies [3]. Figure 1 shows a sintered powder steel cylinder. From the 3D reconstructed dataset (inset), a virtual 2D cross-section (left) reveals several regions of non-sintered volume. In addition to the non-sintered volume, several micron-size cracks were discovered.

Through the incorporation of post-sample optical magnification detector technology, sub-micron XRM has recently extended the application scope of laboratory-based microCT by extending resolution and contrast. As illustrated in Figure 1, interior (local) tomography is routinely performed, as ZEISS Xradia Versa XRM (Figure 2) architecture has been designed to maintain sub-micron spatial resolution for a variety of working distances and sample sizes/geometries. System is capable of imaging at high resolution at large working distance, opening up opportunities for interior tomography of large samples and in situ experiments with large environmental cells. Furthermore, new contrast modes have emerged based on dual energy absorption or diffraction.

Soft materials, such biocompatible photopolymers, consistently pose challenges in generating contrast 
by several techniques, X-ray absorption included. We demonstrate the application of phase contrast techniques on such materials. The tunable contrast enhancement mechanism (propagation-based) within combines optimized absorption contrast, for enhanced imaging of soft materials (low-Z) such as scaffold or implant materials. Porosity is these materials is often engineered. [5]

X-ray microscopes provide internal structural information critical to the evaluation of additive manufacturing materials as well as aid in the design and processing of next generation materials. The ability to visualize structures in 3D with good contrast and resolution even down to 50nm, particularly under operating conditions or elapsed time (4D), can help quickly determine why certain structures, synthesis methods, or operating conditions fail and why others succeed. This can help to bypass some of the empiricism and interpretation that can be associated with less direct structural characterization tools, expediting the iterative design process for the researcher and saving time in the design of future materials.

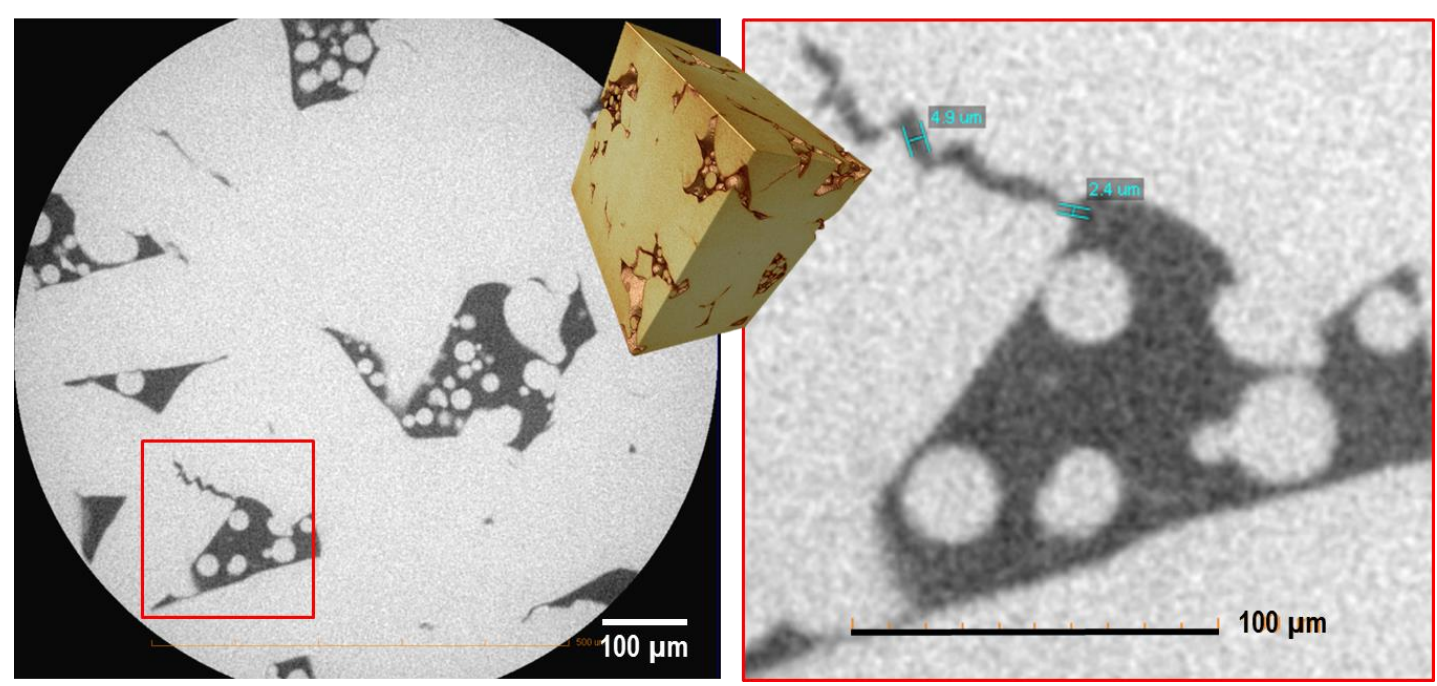

Figure 1 From the 3D reconstructed dataset (inset), a virtual 2D cross-section (left) reveals several regions of non-sintered powder steel volume. In addition to the non-sintered volume, several micronsize cracks were discovered. Sample courtesy of NIST.

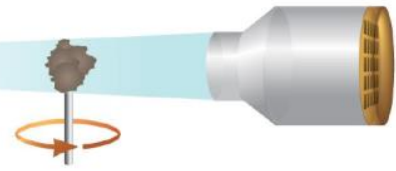

Figure 2 Sub-micron XRM (ZEISS Xradia Versa) optical architecture. Magnification is achieved through a combination of geometric (sample, source, detector placement) and optical (post-sample, variable scintillator-lens-ccd coupling) methods.

\section{References:}

[1] A. Merkle and J. Gelb. Microscopy Today 21, (2013) pp. 10-15.

[2] A. Tkachuk, et al., Z. Kristallogr. 222, (2007) pp. 650-655.

[3] Slotwinski, J. et al. J. Res. Natl. Inst. Stan. 119, (2014) pp. 494-528.

[4] Wen, L. et al. J. Exp Biol 217, (2014) pp. 1656-1666.

[5] Sai, H. et al. Science 341, (2013) pp. 530-534. 